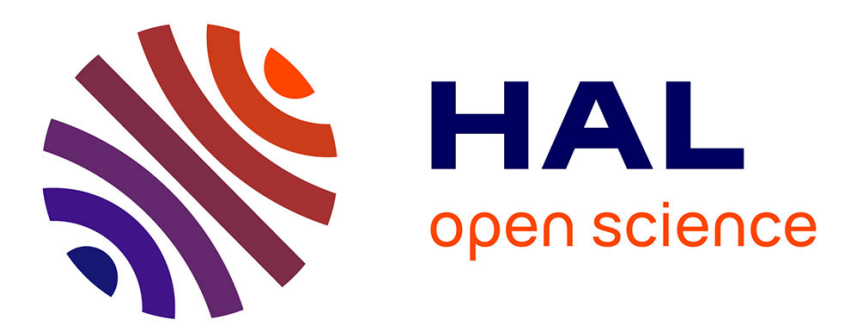

\title{
Plane Lorentzian and Fuchsian hedgehogs
}

Yves Martinez-Maure

\section{To cite this version:}

Yves Martinez-Maure. Plane Lorentzian and Fuchsian hedgehogs. 2014. hal-01054386

\section{HAL Id: hal-01054386 \\ https://hal.science/hal-01054386}

Preprint submitted on 6 Aug 2014

HAL is a multi-disciplinary open access archive for the deposit and dissemination of scientific research documents, whether they are published or not. The documents may come from teaching and research institutions in France or abroad, or from public or private research centers.
L'archive ouverte pluridisciplinaire HAL, est destinée au dépôt et à la diffusion de documents scientifiques de niveau recherche, publiés ou non, émanant des établissements d'enseignement et de recherche français ou étrangers, des laboratoires publics ou privés. 


\title{
Plane Lorentzian and Fuchsian hedgehogs
}

\author{
Yves Martinez-Maure \\ yves.martinez-maure@imj-prg.fr
}

\begin{abstract}
Parts of the Brunn-Minkowski theory can be extended to hedgehogs, which are envelopes of families of affine hyperplanes parametrized by their Gauss map. In $[\mathrm{F}]$, F. Fillastre introduced Fuchsian convex bodies, which are the closed convex sets of Lorentz-Minkowski space that are globally invariant under the action of a Fuchsian group. In this paper, we undertake a study of plane Lorentzian and Fuchsian hedgehogs. In particular, we prove the Fuchsian analogues of classical geometrical inequalities (analogues which are reversed as compared to classical ones).
\end{abstract}

\section{Contents:}

1. Introduction

1.1. Plane Euclidean hedgehogs

1.2. Plane Lorentzian hedgehogs

1.3. Plane Fuchsian hedgehogs

2. Preliminaries

3. Evolute

3.1. Evolute of a spacelike hedgehog $S_{h}$ of $L^{2}$

3.2. Timelike hedgehogs of $L^{2}$ and their evolutes

3.3. Second evolute

4. Duality

5. Convolution

6. Isometric excess and area of the evolute

7. Reversed Bonnesen inequality

2010 MSC: 52A40, 52A55, 53A04, 53B30;

Key words and phrases: Fuchsian and Lorentzian hedgehogs, evolute, duality, convolution, reversed isoperimetric inequality, reversed Bonnesen inequality 


\section{Introduction}

Our main results consist in the Fuchsian analogues of some classical geometrical inequalities. These new results will be presented in Subsection 1.3. For the convenience of the reader, we begin by recalling briefly some definitions and results concerning plane Euclidean hedgehogs in Subsection 1.1. Finally, Subsection 1.2 is devoted to a short introduction of plane Lorentzian hedgehogs and first results concerning evolutes and duality in the Lorentz-Minkowski plane $L^{2}$.

\subsection{Plane Euclidean hedgehogs}

In the Euclidean plane $\mathbb{R}^{2}$, a hedgehog is the envelope of a family of cooriented lines $L(\theta)$ parametrized by the oriented angle $\theta \in \mathbb{S}^{1} \simeq \mathbb{R} / 2 \pi \mathbb{Z}$ from $e_{1}=(1,0)$ to their coorienting normal vector $u(\theta)=(\cos , \sin \theta)$. These cooriented lines $L(\theta)$ have equations

$$
\langle x, u(\theta)\rangle=h(\theta),
$$

where $\langle.,$.$\rangle is the usual inner product on \mathbb{R}^{2}$ and where $h \in C^{1}\left(\mathbb{S}^{1} ; \mathbb{R}\right)$. Partial differentiation of (1) yields

$$
\left\langle x, u^{\prime}(\theta)\right\rangle=h^{\prime}(\theta) .
$$

From (1) and (2), the parametrization of the corresponding hedgehog is

$$
x_{h}: \mathbb{S}^{1} \rightarrow \mathbb{R}^{2}, \theta \mapsto h(\theta) u(\theta)+h^{\prime}(\theta) u^{\prime}(\theta) .
$$

This envelope $\mathcal{H}_{h}:=x_{h}\left(\mathbb{S}^{1}\right)$ is called the (Euclidean) hedgehog with support function $h$. If $h$ is only $C^{1}$ then $\mathcal{H}_{h}$ may be a fractal curve [Y1]. In this paper, we shall be mainly interested in $C^{2}$-hedgehogs, that is, hedgehogs with a $C^{2}$-support function. Note that regular $C^{2}$-hedgehogs of $\mathbb{R}^{2}$ are strictly convex smooth curves and that $C^{2}$-hedgehogs can be regarded as the Minkowski differences of two such convex curves [Y6].

H. Geppert was the first to introduce hedgehogs in $\mathbb{R}^{2}$ and $\mathbb{R}^{3}$ (under the German names stützbare Bereiche in $\mathbb{R}^{2}$ and stützbare Flächen $\mathbb{R}^{3}$ ) in an attempt to extend certain parts of the Brunn-Minkowski theory $[G]$. Many classical inequalities for convex curves find their counterparts in the setting of hedgehogs. Of course, a few adaptations are necessary. In particular, lengths and areas have to be replaced by their algebraic versions. For instance, Theorem 1 extends the classical isoperimetric inequality and gives an upper bound of the isoperimetric deficit in terms of signed area of the evolute. 
Theorem 1 ([Y, Prop. 6]). For any $h \in C^{3}\left(\mathbb{S}^{1} ; \mathbb{R}\right)$, we have:

$$
0 \leq l(h)^{2}-4 \pi a(h) \leq-4 \pi a\left(h^{\prime}\right)
$$

where $l(h)$ and $a(h)$ are respectively the signed length and area of $\mathcal{H}_{h}$ and where $a\left(h^{\prime}\right)$ is the area of its evolute. In each inequality of $(3)$, the equality holds if, and only if, $\mathcal{H}_{h}$ is a circle.

For a recent study of hedgehogs in the Euclidean plane, we refer the reader to $[\mathrm{Y} 6]$.

\subsection{Plane Lorentzian hedgehogs}

In this paper, we shall undertake a similar study replacing the Euclidean plane $\mathbb{R}^{2}$ by the Lorentzian plane $L^{2}$ and the unit circle $\mathbb{S}^{1}$ of $\mathbb{R}^{2}$ by the hyperbolic line $\mathbb{H}^{1}$. In the Lorentzian plane $L^{2}$, a spacelike hedgehog is similarly defined to be the envelope of a family of cooriented spacelike lines $L(t)$ parametrized by the oriented hyperbolic angle $t \in \mathbb{H}^{1} \simeq \mathbb{R}$ from $e_{2}=(0,1)$ to their coorienting normal vector $v(t)=(\sinh t, \cosh t)$, (see Section 2). These cooriented lines $L(t)$ have equations

$$
\langle x, v(t)\rangle_{L}:=h(t),
$$

where $\langle x, y\rangle_{L}:=x_{1} y_{1}-x_{2} y_{2}$ is the Lorentzian inner product of the vectors $x=\left(x_{1}, x_{2}\right)$ and $y=\left(y_{1}, y_{2}\right)$ in $L^{2}$, and where $h \in C^{1}(\mathbb{R} ; \mathbb{R})$. Note that $h(t)$ is the signed distance from the origin to the support line with coorienting unit normal $v(t)$. Partial differentiation of (4) yields

$$
\left\langle x, v^{\prime}(t)\right\rangle_{L}:=h^{\prime}(t) .
$$

From (4) and (5), the parametrization of the corresponding hedgehog is

$$
x_{h}: \mathbb{H}^{1} \rightarrow L^{2}, t \mapsto h^{\prime}(t) v^{\prime}(t)-h(t) v(t) .
$$

This envelope $\mathcal{S}_{h}:=x_{h}\left(\mathbb{H}^{1}\right)$ is called the spacelike hedgehog of $L^{2}$ with support function $h \in C^{1}\left(\mathbb{H}^{1} ; \mathbb{R}\right)$. As in the Euclidean case, we shall generally restrict to $C^{2}$-hedgehogs (i.e., with a $C^{2}$-support function). In Section 3, we shall give a study of their evolutes. In particular, we shall prove the following.

Theorem. For any $h \in C^{3}(\mathbb{R} ; \mathbb{R})$, the second evolute of $\mathcal{S}_{h}$ is the spacelike hedgehog with support function $h^{\prime \prime}$ :

$$
\mathcal{D}\left(\mathcal{D}\left(\mathcal{S}_{h}\right)\right)=\mathcal{S}_{h^{\prime \prime}}
$$


where $\mathcal{D}(\mathcal{C})$ denotes the evolute of a curve $\mathcal{C} \subset L^{2}$ with no inflexion or lightlike point.

In Section 3, we shall also introduce timemike hedgehogs of $L^{2}$ and, in Section 4, we shall show that there is a duality relationship between spacelike hedgehogs and timelike hedgehogs.

\subsection{Plane Fuchsian hedgehogs}

Of course, a spacelike hedgehog $\mathcal{S}_{h} \subset L^{2}$ has no reason to be a compact curve. So, in order to develop a Brunn-Minkowski theory, we are going to replace $\mathbb{H}^{1}$ by its quotient by a Fuchsian group $\Gamma$. In other words: $(i)$ we identify

$$
S O(1,1)=\left\{M=\left(\begin{array}{l}
x_{2} x_{1} \\
x_{1} x_{2}
\end{array}\right) \in M_{2}(\mathbb{R}) \mid x_{2}^{2}-x_{1}^{2}=1\right\}
$$

with the hyperbola

$$
H=\left\{\left(x_{1}, x_{2}\right) \in L^{2} \mid x_{2}^{2}-x_{1}^{2}=1\right\} ;
$$

(ii) we take the subgroup $\Gamma$ of $S O(1,1)$ generated by $(\sinh T, \cosh T) \in$ $\mathbb{H}^{1}=\left\{\left(x_{1}, x_{2}\right) \in H \mid x_{2}>0\right\}$ for some $T \in \mathbb{R}_{+}^{*}$; and (iii) we replace $\mathbb{H}^{1}$ by $\mathbb{H}^{1} / \Gamma \simeq \mathbb{R} / T \mathbb{Z}$. In practice, any $h \in C^{1}\left(\mathbb{H}^{1} / \Gamma ; \mathbb{R}\right)$ will be regarded as a $T$-periodic funtion $h: \mathbb{R} \longmapsto \mathbb{R}$ of class $C^{1}$. The $\Gamma$-hedgehog with support function $h \in C^{1}\left(\mathbb{H}^{1} / \Gamma ; \mathbb{R}\right)$ is then defined to be the curve $\Gamma_{h}$ parametrized by

$$
\gamma_{h}: \mathbb{R} \rightarrow L^{2}, t \mapsto h^{\prime}(t) v^{\prime}(t)-h(t) v(t) .
$$

Note that, for any $t \in \mathbb{R}$, we have $\gamma_{h}(t+T)=g(T)\left[\gamma_{h}(t)\right]$, where $g(T)$ denotes the linear isometry of $L^{2}$ whose matrix in the canonical basis is

$$
\left(\begin{array}{cc}
\cosh T & \sinh T \\
\sinh T & \cosh T
\end{array}\right) .
$$

For every $h \in C^{2}\left(\mathbb{H}^{1} / \Gamma ; \mathbb{R}\right)$, the $C^{1}$-curve $\gamma_{h}:[0, T] \rightarrow L^{2} / \Gamma$, $t \mapsto h^{\prime}(t) v^{\prime}(t)-h(t) v(t)$ is rectifiable and its length is given by

$$
L(h):=\int_{0}^{T}\left\|x_{h}^{\prime}(t)\right\|_{L} d t,
$$

where $\|x\|_{L}:=\sqrt{|\langle x, x\rangle|_{L}}$ for all $x \in L^{2}$. Note that $x_{h}^{\prime}=R_{h} v^{\prime}$, where $R_{h}:=h^{\prime \prime}-h$ is the so-called curvature function of $\Gamma_{h}$. Therefore

$$
L(h):=\int_{0}^{T}\left|R_{h}(t)\right| d t .
$$


If in this last integral we remove the absolute value to take into account the sign of the curvature function of $\Gamma_{h}$, we obtain the so-called algebraic (or signed) length of $\Gamma_{h}$, which is thus given by

$$
l(h):=\int_{0}^{T} R_{h}(t) d t=-\int_{0}^{T} h(t) d t .
$$

Given any $h \in C^{2}\left(\mathbb{H}^{1} / \Gamma ; \mathbb{R}\right)$, let $\Delta_{h}$ be the oriented closed curve of $L^{2}$ consisting of the oriented line segment joining the origin to $\gamma_{h}(0)$, followed by the oriented curve $\Gamma_{h}$ and finally the oriented line segment joining $\gamma_{h}(T)$ to the origin. Denote by $\left(\Delta_{h}\right)^{-}$the curve obtained from $\Delta_{h}$ by taking the opposite orientation (see Figure 1). Define the algebraic (or signed) area of the $\Gamma$-hedgehog $\Gamma_{h}$ to be the algebraic area bounded by $\left(\Delta_{h}\right)^{-}$that is,

$$
a(h):=\int_{L^{2}} i_{h}(x) d \lambda(x),
$$

where $\lambda$ the Lebesgue measure, $i_{h}(x)$ the winding number of $x$ with respect to $\left(\Delta_{h}\right)^{-}$for $x \in L^{2}-\left(\Delta_{h}\right)^{-}$, and $i_{h}(x)=0$ for $x \in\left(\Delta_{h}\right)^{-}$. An easy straightforward calculation gives

$a(h)=\frac{1}{2} \int_{\left(\Delta_{h}\right)^{-}} x_{1} d x_{2}-x_{2} d x_{1}=\frac{1}{2} \int_{0}^{T} h(t) R_{h}(t) d t=\frac{1}{2} \int_{0}^{T}\left(h^{2}+\left(h^{\prime}\right)^{2}\right)(t) d t$. 


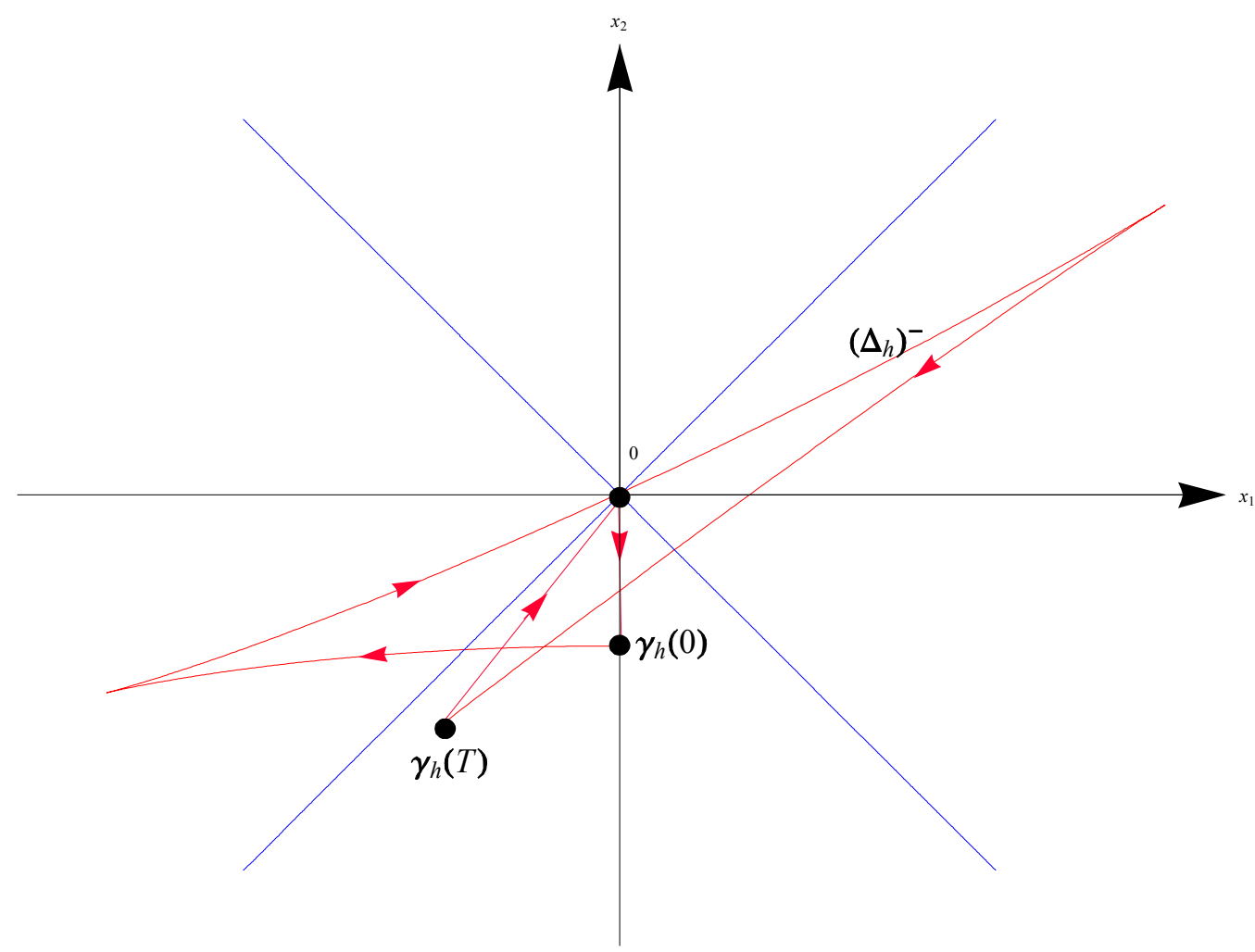

Figure 1. The oriented closed curve $\left(\Delta_{h}\right)^{-}$when $h(t):=1+\cos (2 \pi t)$

In the Fuchsian case, many geometric inequalities will be reversed. A first example is given by the following obvious result.

Proposition. The map $\sqrt{a}: C^{2}\left(\mathbb{H}^{1} / \Gamma ; \mathbb{R}\right) \rightarrow \mathbb{R}_{+}, h \longmapsto \sqrt{a(h)}$ is a norm associated with a scalar product $(h, k) \longmapsto a(h, k)$. In particular, for any $(h, k) \in C^{2}\left(\mathbb{H}^{1} / \Gamma ; \mathbb{R}\right)^{2}$, we have

$$
\sqrt{a(h+k)} \leq \sqrt{a(h)}+\sqrt{a(k)}
$$

and

$$
a(h, k)^{2} \leq a(h) a(k),
$$

with equalities if, and only if, $\Gamma_{h}$ and $\Gamma_{k}$ are homothetic (here, "homothetic" means that there exists $(\lambda, \mu) \in \mathbb{R}^{2}-\{(0,0)\}$ such that $\left.\lambda h+\mu k=0\right)$.

Indeed, Inequality (6) (resp. (7)) has to be compared with the BrunnMinkowski inequality (resp. Minkowski inequality) in $\mathbb{R}^{2}$ (e.g., see $[\mathrm{S}$, 
Section 7$])$ : for any pair $(H, K)$ of convex bodies of $\mathbb{R}^{2}$, we have

$$
\sqrt{a(H+K)} \geq \sqrt{a(H)}+\sqrt{a(K)}
$$

and

$$
a(H, K)^{2} \geq a(H) a(K),
$$

where $a(L)$ (resp. $a(H, K))$ is the area (resp. the mixed area) of $L$ (resp. $(H, K))$. By taking $k=-1$ (that is, $\Gamma_{k}=\mathbb{H}^{1}$ ) in $(7)$, we obtain the following reversed isoperimetric inequality

$$
a(h) \geq \frac{l(h)^{2}}{2 T},
$$

with equality if, and only if, $\Gamma_{h}$ and $\mathbb{H}^{1}$ are homothetic (that is, $h$ is constant). In Section 6, we shall prove an other reversed geometric inequality given by the following analogous of Theorem 1 for Fuchsian hedgehogs.

Theorem 2. Let $T \in] 0,2 \pi]$. For any $T$-periodic function $h: \mathbb{R} \rightarrow \mathbb{R}$ of class $C^{3}$, we have:

$$
0 \leq 2 T a(h)-l(h)^{2} \leq 2 T a\left(h^{\prime}\right),
$$

where $l(h)$ and $a(h)$ are respectively the signed length and area of $\Gamma_{h}$ and $a\left(h^{\prime}\right)$ the area of its evolute.

Note that $2 T a(h)-l(h)^{2}$ provides a measure of how far $\Gamma_{h}$ deviates from a $\Gamma$-hedgehog given by a spacelike branch of a hyperbola. A lower bound of the isoperimetric excess $a(h)-l(h)^{2} / 2 T$ is given by the following reversed Bonnesen inequality, which we shall prove in Section 7.

Theorem 3 (Reversed Bonnesen inequality). For any T-periodic function $h: \mathbb{R} \rightarrow \mathbb{R}$ of class $C^{2}$, we have:

$$
\frac{1}{2 T}(R-r)^{2} \leq a(h)-\frac{l(h)^{2}}{2 T},
$$

where $l(h)$ and $a(h)$ are respectively the signed length and area of $\Gamma_{h}$ and where $r:=\min _{0 \leq t \leq T}(-h(t))$ and $R:=\max _{0 \leq t \leq T}(-h(t))$. Furthermore, the equality holds if and only if $R=r$.

Recall that Bonnesen's sharpening of the isoperimetric inequality for a convex body $K$ with non-empty interior in $\mathbb{R}^{2}$ reads as follows: 


$$
L^{2}-4 \pi A \geq \pi^{2}(R-r)^{2},
$$

where $L$ and $A$ are respectively the perimeter and the area of $K$ and where $r$ and $R$ stand respectively for the inradius and the circumradius of $K$ (e.g., see [E, pp. 108-110]).

Acknowledgement. I would like to thank F. Fillastre for sending me a preliminary version of his paper $[\mathrm{F}]$. It did not contain any notion of hedgehog but I immediately understood that existence of Fuchsian hedgehogs was a direct by-product of Fillastre's work and I made him the remark in an e-mail dated 2011-11-17. I would like also thank F. Fillastre for stimulative conversations during the preparation of this paper.

\section{Preliminaries}

The Lorentzian plane $L^{2}$ is the vector space $\mathbb{R}^{2}$ endowed with the pseudoscalar product $\langle x, y\rangle_{L}:=x_{1} y_{1}-x_{2} y_{2}$, for any $x=\left(x_{1}, x_{2}\right)$ and $y=\left(y_{1}, y_{2}\right)$. For any $x \in L^{2}$, define the norm of $x$ by $\|x\|_{L}=\sqrt{\left|\langle x, x\rangle_{L}\right|}$ and the sign of $x$ by $\varepsilon(x)=\operatorname{sgn}\left(\langle x, x\rangle_{L}\right)$, where $\operatorname{sgn}$ denotes the sign function: $\operatorname{sgn}(t)$ is 1,0 , or -1 if $t$ is positive, zero, or negative, respectively. A nonzero vector $x \in L^{2}$ is said to be spacelike if $\varepsilon(x)=1$, lightlike if $\varepsilon(x)=0$ and timelike if $\varepsilon(x)=-1$. Let $e_{2}=(0,1)$. A timelike vector $x=\left(x_{1}, x_{2}\right) \in L^{2}$ is said to be a future vector if $\left\langle x, e_{2}\right\rangle_{L}<0$, that is, if $x_{2}>0$. We shall denote by $F$ the set of all future timelike vectors:

$$
F=\left\{x=\left(x_{1}, x_{2}\right) \in L^{2} \mid\langle x, x\rangle_{L}<0 \text { and } x_{2}>0\right\} .
$$

The hyperbolic line $\mathbb{H}^{1}$ is the set of all unit future timelike vectors:

$$
\mathbb{H}^{1}:=\left\{x=\left(x_{1}, x_{2}\right) \in L^{2} \mid\langle x, x\rangle_{L}=-1 \text { and } x_{2}>0\right\} .
$$

In other words, $\mathbb{H}^{1}$ is the upper branch of the hyperbola $x_{2}^{2}=x_{1}^{2}+1$. It will play in $L^{2}$ the same role as the one the unit circle $\mathbb{S}^{1}$ plays in the Euclidean plane $\mathbb{R}^{2}$. For any $t \in \mathbb{R}$, let $g(t)$ be the linear isometry of the Lorentzian plane whose matrix in the canonical basis of $\mathbb{R}^{2}$ is

$$
\left(\begin{array}{ll}
\cosh t & \sinh t \\
\sinh t & \cosh t
\end{array}\right)
$$

These isometries $g(t)$ constitute the group $G$ of hyperbolic translations of $L^{2}$. Note that $G$ is an abelian subgroup of $O(1,1)$ and that $g: \mathbb{R} \rightarrow G$, $t \mapsto g(t)$ is a group isomorphism: $g(s+t)=g(s) g(t)$ for all $s, t \in \mathbb{R}$. The hyperbolic line $\mathbb{H}^{1}$ can be regarded as the orbit of $e_{2}$ under the action of $G$. Any $v(t)=(\sinh t, \cosh t) \in \mathbb{H}^{1}$ is identified with the unique $t \in \mathbb{R}$ 
such that $g(t)\left(e_{2}\right)=v(t)$. For any $x, y \in \mathbb{H}^{1}$, the oriented hyperbolic angle from $x$ to $y$ is the unique $t$ such that $g(t)(x)=y$.

A (smooth) curve of $L^{2}$ is a differentiable map $c: I \subset \mathbb{R} \rightarrow L^{2}$, where $I$ is an open interval. A curve $c: I \rightarrow L^{2}$ is said to be regular at $t$ if $c^{\prime}(t) \neq 0$. The curve is said to be regular if it is regular at every $t \in I$. A curve $c: I \rightarrow L^{2}$ is said to be spacelike (resp. lightlike, timelike) at $t$ if $c^{\prime}(t)$ is a spacelike (resp. null or lightlike, timelike) vector. The curve is said to be spacelike (resp. timelike) if it is spacelike (resp. timelike) at every $t \in I$.

Let $\sigma$ be the anti-isometric involutive operator of $L^{2}$ that is given by $\sigma\left(x_{1}, x_{2}\right)=\left(x_{2}, x_{1}\right)$ for all $\left(x_{1}, x_{2}\right) \in L^{2}$. For any nonzero vector $x$ of $L^{2}$, let $x^{\perp}:=\varepsilon(x) \sigma(x)$. Note that, for any $x \in L^{2}-\{(0,0)\},\left(x, x^{\perp}\right)$ is a positively oriented basis of $L^{2}$ endowed with the orientation of the canonical basis of $\mathbb{R}^{2}$.

Let $c: I \rightarrow L^{2}$ be a spacelike (resp. timelike) curve of class $C^{2}$. At any point of $c: I \rightarrow L^{2}$, we can define the oriented Frenet frame $(T(t), N(t))$ consisting of Frenet vectors

$$
T(t):=\frac{c^{\prime}(t)}{\left\|c^{\prime}(t)\right\|_{L}} \quad \text { and } \quad N(t):=T(t)^{\perp} .
$$

If $c: I \rightarrow L^{2}$ is parametrized by the pseudo arc length $s$ (that is, if $\left\|c^{\prime}(s)\right\|_{L}=1$ for all $\left.s \in I\right)$, then the algebraic curvature of $c$ is defined to be the function $\kappa$ such that $T^{\prime}(s)=\kappa(s) N(s)$. If it is not the case, a straightforward computation using the fact that $d s / d t=\left\|c^{\prime}(t)\right\|_{L}$ shows that the algebraic curvature is given by

$$
\kappa(t):=\frac{\left\langle c^{\prime}(t), \sigma\left(c^{\prime \prime}(t)\right)\right\rangle_{L}}{\left\|c^{\prime}(t)\right\|_{L}^{3}} .
$$

If $c: I \rightarrow L^{2}$ is a spacelike hedgehog $x_{h}: \mathbb{H}^{1} \rightarrow L^{2}$ with support function $h \in C^{3}(\mathbb{R} ; \mathbb{R})$, then $c^{\prime}=x_{h}^{\prime}=R_{h} v^{\prime}$, where $R_{h}:=h^{\prime \prime}-h$ is the so-called curvature function of $\mathcal{S}_{h}$. In this case, we hence obtain

$$
T=\operatorname{sgn}\left(R_{h}\right) v^{\prime}, \quad N=\operatorname{sgn}\left(R_{h}\right) v \quad \text { and } \quad \kappa(t)=\frac{1}{\left|R_{h}\right|} .
$$

\section{Evolute}

\subsection{Evolute of a spacelike hedgehog $\mathcal{S}_{h}$ of $L^{2}$}

In this subsection, $h$ will denote any $C^{3}$-function from $\mathbb{R}$ to $\mathbb{R}$. As in the Euclidean case, the evolute of the spacelike hedgehog $\mathcal{S}_{h}$ can be defined in two different but equivalent ways: as an envelope or as a locus. 


\subsubsection{Evolute of $\mathcal{S}_{h}$ as the envelope of its normal lines}

For every $t \in \mathbb{R}$, the support line $L_{h}(t)$, with coorienting unit normal vector $v(t):=(\sinh t, \cosh t)$, has equation

$$
\langle x, v(t)\rangle_{L}:=h^{\prime}(t) .
$$

Let $N_{h}(t)$ be the line through $x$ that is orthogonal to $L_{h}(t)$ in $L^{2}$. We shall say that $N_{h}(t)$ is a normal line to $\mathcal{H}_{h}$ at $x_{h}(t)$. This normal line $N_{h}(t)$ has equation

$$
\langle x, v(t)\rangle_{L}:=h^{\prime \prime}(t) .
$$

Define the evolute $\mathcal{D}\left(\mathcal{S}_{h}\right)$ of the spacelike hedgehog $\mathcal{S}_{h} \subset L^{2}$ to be the envelope of the family $\left(N_{h}(t)\right)_{t \in \mathbb{R}}$ of its normal lines. This evolute $\mathcal{D}\left(\mathcal{S}_{h}\right)$ is thus the curve of $L^{2}$ parametrized by

$$
c_{h}: \mathbb{R} \rightarrow L^{2}, t \longmapsto c_{h}(t),
$$

where $c_{h}(t)$ is the unique solution of the system

$$
\left\{\begin{array}{l}
\langle x, v(t)\rangle_{L}:=h^{\prime}(t) \\
\langle x, v(t)\rangle_{L}:=h^{\prime \prime}(t)
\end{array},\right.
$$

that is, $c_{h}(t)=h^{\prime}(t) v^{\prime}(t)-h^{\prime \prime}(t) v(t)$.

\subsubsection{Evolute of $\mathcal{S}_{h}$ as the locus of its centers of curvature}

The evolute $\mathcal{D}\left(\mathcal{S}_{h}\right)$ of the spacelike hedgehog $\mathcal{S}_{h} \subset L^{2}$ can also be defined as the locus of all its centers of curvature. First note that

$$
x_{h}^{\prime}(t)=R_{h}(t) v^{\prime}(t),
$$

for all $t \in \mathbb{H}^{1} \simeq \mathbb{R}$. Since $\mathcal{S}_{h}:=x_{h}\left(\mathbb{H}^{1}\right)$ is an envelope parametrized by its coorienting unit normal vector field, the center of curvature of $\mathcal{S}_{h}$ at $x_{h}(t)$ is defined to be

$$
c_{h}(t):=x_{h}(t)-R_{h}(t) v(t)
$$

that is

$$
c_{h}(t)=h^{\prime}(t) v^{\prime}(t)-h^{\prime \prime}(t) v(t),
$$

for all $t \in \mathbb{H}^{1} \simeq \mathbb{R}$. Of course, if $x_{h}$ is regular at $t$ then

$$
c_{h}(t)=x_{h}(t)-\frac{N(t)}{\kappa(t)},
$$

but the center of curvature $c_{h}(t)$ is well-defined even if $x_{h}^{\prime}(t)=0$. 


\subsection{Timelike hedgehogs of $L^{2}$ and their evolutes}

\subsubsection{Definitions}

We can also define timelike hedgehogs of $L^{2}$. The timelike hedgehog with support function $h \in C^{1}(\mathbb{R} ; \mathbb{R})$ is defined to be the envelope $\mathcal{T}_{h}$ of the family $\left(L_{h}^{\prime}(t)\right)_{t \in \mathbb{R}}$ of cooriented timelike lines with equation

$$
\left\langle x, v^{\prime}(t)\right\rangle_{L}:=h(t),
$$

$v^{\prime}(t)=(\cosh t, \sinh t)$ being the unit coorienting normal vector of $L_{h}^{\prime}(t)$. Partial differentiation of (7) yields

$$
\langle x, v(t)\rangle_{L}:=h^{\prime}(t) .
$$

From (7) and (8), the parametrization of the timelike hedgehog $\mathcal{T}_{h}$ is

$$
y_{h}: \mathbb{R} \rightarrow L^{2}, t \mapsto h(t) v^{\prime}(t)-h^{\prime}(t) v(t) .
$$

Note that for every $t \in \mathbb{R}$, we have

$$
y_{h}^{\prime}(t)=-R_{h}(t) v(t) .
$$

The evolute $\mathcal{D}\left(\mathcal{T}_{h}\right)$ of a timelike hedgehog $\mathcal{T}_{h} \subset L^{2}$ is defined to be the envelope of the family of its normal lines (i.e., the envelope of the family of lines with equation $\left.\langle x, v(t)\rangle_{L}:=h^{\prime}(t)\right)$ or, equivalently, the locus of its centers of curvature

$$
d_{h}(t):=y_{h}(t)-\left(-R_{h}(t) v^{\prime}(t)\right)=h^{\prime \prime}(t) v^{\prime}(t)-h^{\prime}(t) v(t),
$$

$(t \in \mathbb{R})$.

\subsubsection{Relationship between $\mathcal{S}_{h}=x_{h}(\mathbb{R})$ and $\mathcal{T}_{h}=y_{h}(\mathbb{R})$}

Let $\Sigma$ be the anti-isometric involutive operator of $L^{2}$ that is given by $\Sigma(x)=-\sigma(x)$ for all $x \in L^{2}$. Note that $\Sigma \circ v=-v^{\prime}$ and $\Sigma \circ v^{\prime}=-v$.

Proposition. For any $h \in C^{1}(\mathbb{R} ; \mathbb{R})$, the spacelike hedgehog $\mathcal{S}_{h}$ and the timelike hedgehog $\mathcal{T}_{h}$ are related by

$$
\mathcal{T}_{h}=\Sigma\left(\mathcal{S}_{h}\right) \quad \text { and } \quad \mathcal{S}_{h}=\Sigma\left(\mathcal{T}_{h}\right) .
$$

Proof. Indeed, their respective parametrizations $x_{h}:=h^{\prime} v^{\prime}-h v$ and $y_{h}:=h v^{\prime}-h^{\prime} v$ are such that $y_{h}=\Sigma \circ x_{h}$ and $x_{h}=\Sigma \circ y_{h}$. 


\subsection{Second evolute}

Proposition. For any $h \in C^{2}(\mathbb{R} ; \mathbb{R})$, the evolute of the spacelike hedgehog $\mathcal{S}_{h}$ (resp. of the timelike hedgehog $\mathcal{T}_{h}$ ) can be given by

$$
\mathcal{D}\left(\mathcal{S}_{h}\right)=\Sigma\left(\mathcal{S}_{h^{\prime}}\right) \quad\left(\text { resp. } \mathcal{D}\left(\mathcal{T}_{h}\right)=\Sigma\left(\mathcal{T}_{h^{\prime}}\right)\right)
$$

and hence by

$$
\mathcal{D}\left(\mathcal{S}_{h}\right)=\mathcal{T}_{h^{\prime}} \quad\left(\text { resp. } \mathcal{D}\left(\mathcal{T}_{h}\right)=\mathcal{S}_{h^{\prime}}\right)
$$

from the previous proposition.

Proof. Indeed, $c_{h}:=h^{\prime} v^{\prime}-h^{\prime \prime} v$ (resp. $d_{h}:=h^{\prime \prime} v^{\prime}-h^{\prime} v$ ) satisfies $\Sigma \circ c_{h}=-h^{\prime} v+h^{\prime \prime} v^{\prime}=x_{h^{\prime}}$ (resp. $\left.\Sigma \circ d_{h}=-h^{\prime \prime} v+h^{\prime} v^{\prime}=y_{h^{\prime}}\right)$ and hence $c_{h}=\Sigma \circ x_{h^{\prime}}\left(\right.$ resp. $\left.d_{h}=\Sigma \circ y_{h^{\prime}}\right)$.

See Figure 2 for an illustration.

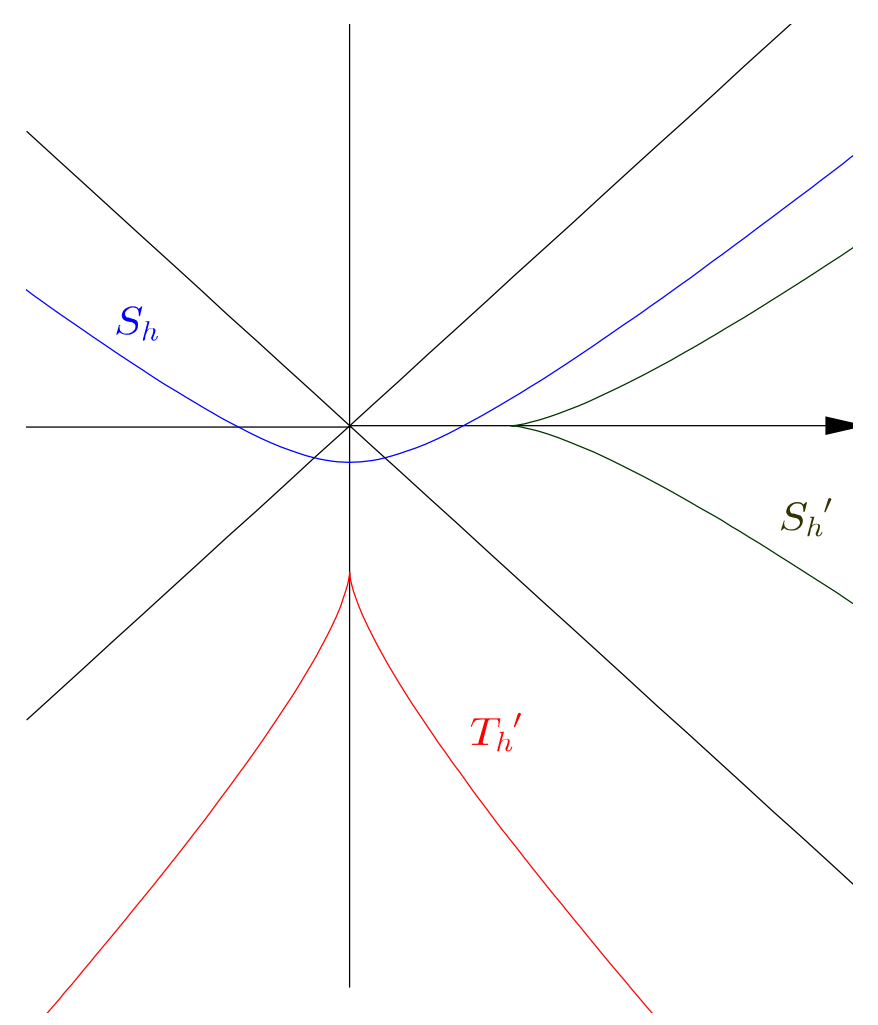

Figure $2 . \mathcal{S}_{h}$ and its evolute $\mathcal{T}_{h^{\prime}}$ if $h(t):=\cosh (2 t)$

A straightforward consequence is the following. 
Corollary. For any $h \in C^{3}(\mathbb{R} ; \mathbb{R})$, the second evolute of the spacelike hedgehog $\mathcal{S}_{h}$ (resp. of the timelike hedgehog $\mathcal{T}_{h}$ ) is simply the spacelike (resp. timelike hedgehog) with support function $h^{\prime \prime}$ :

$$
\mathcal{D}^{2}\left(\mathcal{S}_{h}\right):=\mathcal{D}\left(\mathcal{D}\left(\mathcal{S}_{h}\right)\right)=\mathcal{S}_{h^{\prime \prime}} \quad\left(\text { resp. } \mathcal{D}^{2}\left(\mathcal{T}_{h}\right)=\mathcal{D}\left(\mathcal{D}\left(\mathcal{T}_{h}\right)\right)=\mathcal{T}_{h^{\prime \prime}}\right)
$$

\section{Duality}

Let $c: I \subset \mathbb{R} \rightarrow L^{2}$ be a spacelike or timelike curve of $L^{2}$ and let $p_{c}: I \rightarrow L^{2}$ be its pedal curve: for any $t \in I, p_{c}(t)$ is the foot of the perpendicular from the origin to the tangent line to $c$ at $c(t)$. Note that, replacing tangent lines by support lines, we can define the pedal curve of a spacelike (resp. timelike) hedgehog even if $x_{h}$ (resp. $y_{h}$ ) is not regular. Assume that $\|c(t)\|_{L} \cdot\left\|p_{c}(t)\right\|_{L} \neq 0$ for all $t \in I$. Define the star curve of $c$ to be the curve $c^{*}: I \subset \mathbb{R} \rightarrow L^{2}$ given by $c^{*}:=i \circ p_{C}$, where

$$
i(x):=\varepsilon(x) \frac{x}{\|x\|_{L}^{2}} \quad \text { for all } x \in L^{2} \text { such that }\|x\|_{L} \neq 0,
$$

(recall that $\varepsilon(x):=\operatorname{sgn}\left(\langle x, x\rangle_{L}\right)$ ). If $c: \mathbb{R} \rightarrow L^{2}$ is a spacelike hedgehog $x_{h}$ (resp. a timelike hedgehog $y_{h}$ ), then $p_{c}=-h v$ (resp. $\left.p_{c}=h v^{\prime}\right)$ and, assuming that $h .\left\|x_{h}\right\|_{L} \neq 0$ (resp. $h .\left\|y_{h}\right\|_{L} \neq 0$ ), we can define its star curve in the same way.

Proposition. If $c: \mathbb{R} \rightarrow L^{2}$ is a spacelike hedgehog $x_{h}$ (resp. a timelike hedgehog $\left.y_{h}\right)$, then $\left(c^{*}\right)^{*}=c$.

Proof. If $c=x_{h}$ (resp. $\left.c=y_{h}\right)$, then $p_{c}=-h v\left(\right.$ resp. $\left.p_{c}=h v^{\prime}\right)$. Thus

$$
x_{h}^{*}=\frac{v}{h} \quad\left(\operatorname{resp} \cdot y_{h}^{*}=\frac{v^{\prime}}{h}\right) .
$$

Differentiation gives

$$
\left(x_{h}^{*}\right)^{\prime}=\frac{y_{h}}{h^{2}} \quad\left(\operatorname{resp} . \quad\left(y_{h}^{*}\right)^{\prime}=-\frac{x_{h}}{h^{2}}\right) .
$$

Now

$$
x_{h}^{*}=\frac{h^{\prime} y_{h}-h x_{h}}{h\left(h^{2}-\left(h^{\prime}\right)^{2}\right)} \quad\left(\text { resp. } y_{h}^{*}=\frac{h y_{h}-h^{\prime} x_{h}}{h\left(h^{2}-\left(h^{\prime}\right)^{2}\right)}\right) .
$$

Therefore

$$
p_{x_{h}^{*}}=\frac{x_{h}}{\left(h^{\prime}\right)^{2}-h^{2}} \quad\left(\text { resp. } p_{y_{h}^{*}}=\frac{y_{h}}{h^{2}-\left(h^{\prime}\right)^{2}}\right)
$$


and hence

$$
\left(x_{h}^{*}\right)^{*}=x_{h} \quad\left(\operatorname{resp} . \quad\left(y_{h}^{*}\right)^{*}=y_{h}\right) .
$$

Definition. For any $h \in C^{1}(\mathbb{R} ; \mathbb{R})$ such that $h(t) .\left\|x_{h}(t)\right\|_{L} \neq 0$ (resp. $\left.h(t) .\left\|y_{h}(t)\right\|_{L} \neq 0\right)$ for all $t \in \mathbb{R}$, we shall say that $\mathcal{S}_{h}^{*}:=x_{h}^{*}(\mathbb{R})($ resp. $\mathcal{T}_{h}^{*}:=y_{h}^{*}(\mathbb{R})$ ) is the dual curve of the spacelike (resp. timelike) hedgehog $\mathcal{S}_{h}\left(\right.$ resp. $\left.\mathcal{T}_{h}\right)$.

\section{Convolution}

Differences of convex bodies of the Euclidean plane $\mathbb{R}^{2}$ do not only constitute a real vector space $\left(\mathcal{H}^{2},+,.\right)$ but also a commutative and associative $\mathbb{R}$-algebra. Indeed, as noticed by H. Görtler [G1, G2], we can define the convolution product of two hedgehogs $\mathcal{H}_{f}$ and $\mathcal{H}_{g}$ of $\mathbb{R}^{2}$ as the hedgehog whose support function is given by

$$
(f * g)(\theta)=\frac{1}{2 \pi} \int_{0}^{2 \pi} f(\theta-\alpha) g(\alpha) d \alpha,
$$

for all $\theta \in \mathbb{S}^{1} \simeq \mathbb{R} / 2 \pi \mathbb{Z}$ and we can check at once that $\left(\mathcal{H}^{2},+, ., *\right)$ is then a commutative and associative algebra. The interest is, of course, that the convolution product of two Euclidean hedgehogs inherits many properties of the factors [Y6, Section 6]. In particular, H. Görtler noticed that the convolution product of two plane convex bodies is still a plane convex body. The purpose of the present section is to give a similar result for Fuchsian hedgehogs.

Let $h \in C^{2}\left(\mathbb{H}^{1} ; \mathbb{R}\right)$. Recall that, for all $t \in \mathbb{H}^{1} \simeq \mathbb{R}$, we have:

$$
x_{h}^{\prime}(t)=R_{h}(t) v^{\prime}(t),
$$

where $R_{h}:=h^{\prime \prime}-h$. Therefore, the spacelike hedgehog $\mathcal{S}_{h}=x_{h}\left(\mathbb{H}^{1}\right)$ is a regular curve if, and only if, its curvature function $R_{h}$ is everywhere nonzero. In that case, $\mathcal{S}_{h}$ will be said to be convex.

Definition. Let $h \in C^{2}\left(\mathbb{H}^{1} ; \mathbb{R}\right)$. The spacelike hedgehog $\mathcal{S}_{h}$ is said to be convex if its curvature function $R_{h}:=h^{\prime \prime}-h$ is everywhere nonzero on $\mathbb{H}^{1}$. It is said to be future convex (resp. past convex) if its curvature function is everywhere positive (resp. negative) on $\mathbb{H}^{1}$. 
Definition. Let $h \in C^{2}\left(\mathbb{H}^{1} / \Gamma ; \mathbb{R}\right)$. The $\Gamma$-hedgehog $\Gamma_{h}$ is said to be $a \Gamma$ hedgehog of class $C_{+}^{2}$ of $F=\left\{x=\left(x_{1}, x_{2}\right) \in L^{2} \mid\langle x, x\rangle_{L}<0\right.$ and $\left.x_{2}>0\right\}$ if $h<0$ and $R_{h}>0$.

Remark. A $\Gamma$-hedgehog of class $C_{+}^{2}$ of $F$ can indifferently be regarded as a convex curve of $F$ or as a convex closed curve of $F / \Gamma$.

Definition. Let $\Gamma_{f}$ and $\Gamma_{g}$ be $\Gamma$-hedgehogs whose respective support functions $f$ and $g$ are in $C^{1}\left(\mathbb{H}^{1} / \Gamma ; \mathbb{R}\right)$. The convolution of $\Gamma_{f}$ and $\Gamma_{g}$ is the $\Gamma$-hedgehog $\Gamma_{f * g}$ whose support function is defined by

$$
(f * g)(t)=-\int_{0}^{T} f(t-s) g(s) d s \quad \text { for all } t \in[0, T] .
$$

The operation of convolution of $\Gamma$-hedgehogs is of course commutative, associative and distributive over addition. Here is an analogous result of Görtler's theorem.

Proposition. Let $\Gamma_{f}$ and $\Gamma_{g}$ be $\Gamma$-hedgehogs whose respective support functions $f$ and $g$ are in $C^{2}\left(\mathbb{H}^{1} / \Gamma ; \mathbb{R}\right)$. If $\Gamma_{f}$ is a $\Gamma$-hedgehog of class $C_{+}^{2}$ of $F$ and if $g$ is negative, then $\Gamma_{f * g}$ is a $\Gamma$-hedgehog of class $C_{+}^{2}$ of $F$.

Proof. If $f<0, g<0$ and $R_{h}>0$, then $(f * g)<0$ and $R_{f * g}>0$. Indeed, the first inequality is trivial and

$$
\begin{aligned}
R_{f * g}(t) & =(f * g)^{\prime \prime}(t)-(f * g)(t)=\left(f * g^{\prime \prime}\right)(t)-(f * g)(t) \\
& =\left(f *\left(g^{\prime \prime}-g\right)\right)(t)=\left(f * R_{g}\right)(t)=-\int_{0}^{T} f(t-s) R_{g}(s) d s
\end{aligned}
$$

is positive for all $t \in[0, T]$ since $f<0$ and $R_{g}>0$.

\section{Isometric excess and area of the evolute}

Let us prove the following analogous of Theorem 1 for Fuchsian hedgehogs.

Theorem 2. Let $T \in] 0,2 \pi]$. For any $T$-periodic function $h: \mathbb{R} \rightarrow \mathbb{R}$ of class $C^{3}$, we have:

$$
0 \leq 2 T a(h)-l(h)^{2} \leq 2 T a\left(h^{\prime}\right),
$$

where $l(h)$ and $a(h)$ are respectively the signed length and area of $\Gamma_{h}$ and $a\left(h^{\prime}\right)$ the area of its evolute. 
Proof. The first inequality is simply the isoperimetric inequality (10). Let us prove the second one. First note that:

$$
a(h)-a\left(h^{\prime}\right)=\frac{1}{2}\left(\int_{0}^{T} h^{2} d t-\int_{0}^{T}\left(h^{\prime \prime}\right)^{2} d t\right) .
$$

Let $a_{n}(f)$ and $b_{n}(f)$ denote the Fourier coefficients of a $T$-periodic differentiable function $f: \mathbb{R} \rightarrow \mathbb{R}$ :

$$
\begin{aligned}
& a_{0}(f):=\frac{1}{T} \int_{0}^{T} f(t) d t \\
& a_{n}(f):=\frac{2}{T} \int_{0}^{T} f(t) \cos n \omega t d t \\
& \text { and } b_{n}(f):=\frac{2}{T} \int_{0}^{T} f(t) \sin n \omega t d t
\end{aligned}
$$

where $\omega:=2 \pi / T$ and $n \in \mathbb{N}^{*}$. Recall that $a_{n}\left(h^{\prime \prime}\right)=-(n \omega)^{2} a_{n}(h)$ and $b_{n}\left(h^{\prime \prime}\right)=-(n \omega)^{2} b_{n}(h)$ for all $n \in \mathbb{N}^{*}$. By applying the Parseval equality, we thus obtain

$$
\frac{1}{2} \int_{0}^{T}\left(h^{2}-\left(h^{\prime \prime}\right)^{2}\right)(t) d t=\frac{T}{2} a_{0}(h)^{2}+\frac{T}{4} \sum_{n=1}^{+\infty}\left(1-(n \omega)^{4}\right)\left(a_{n}(h)^{2}+b_{n}(h)^{2}\right) .
$$

Since the sum in the right-hand side is obviously nonpositive, we deduce that

$$
\frac{1}{2} \int_{0}^{T}\left(h^{2}-\left(h^{\prime \prime}\right)^{2}\right)(t) d t \leq \frac{T}{2} a_{0}(h)^{2}=\frac{l(h)^{2}}{2 T}
$$

Therefore

$$
a(h)-\frac{l(h)^{2}}{2 T} \leq a\left(h^{\prime}\right),
$$

which achieves the proof.

Remarks. 1. For $T \in] 0,2 \pi\left[\right.$, the equality $2 T a(h)-l(h)^{2}=2 T a\left(h^{\prime}\right)$ holds if, and only if, $h$ is constant.

2. For $T=2 \pi$, the equality $2 T a(h)-l(h)^{2}=2 T a\left(h^{\prime}\right)$ may hold for nonconstant $h \in C^{3}\left(\mathbb{H}^{1} / \Gamma ; \mathbb{R}\right)$. Consider for instance $h(t):=\cos t$.

3. The assumption $T \in] 0,2 \pi]$ is necessary even if we restrict to $\Gamma$ hedgehogs that are $\Gamma$-hedgehogs of class $C_{+}^{2}$ of $F$. Consider for instance $h(t):=-2+\cos \left(\frac{2 \pi}{7} t\right)$, which is such that $h<0$ and $R_{h}>0$. 


\section{Reversed Bonnesen inequality}

Let $K$ be a convex body with non-empty interior in $\mathbb{R}^{2}$. In the 1920 s, $\mathrm{T}$. Bonnesen gave various sharpening of the classical isoperimetric inequality

$$
A \leq \frac{L^{2}}{4 \pi}
$$

where $L$ and $A$ denote respectively the perimeter and the area of $K$. In particular, he proved the inequality

$$
L^{2}-4 \pi A \geq \pi^{2}(R-r)^{2}
$$

where $r$ and $R$ are respectively the inradius and the circumradius of $K$ (i.e., the radii of the largest inscribed and the smallest circumscribed circles of the boundary of $K$, respectively). He further proved that the equality holds in (12) if and only if $R=r$, i.e., if $K$ is a disc. The proof by Bonnesen is reproduced in [E, pp. 108-110]. For a survey of Bonnesentype inequalities in Euclidean spaces, we refer the reader to [O]. Let us prove the following reversed Bonnesen inequality for Fuchsian hedgehogs.

Theorem 3. For any T-periodic function $h: \mathbb{R} \rightarrow \mathbb{R}$ of class $C^{2}$, we have:

$$
\frac{1}{2 T}(R-r)^{2} \leq a(h)-\frac{l(h)^{2}}{2 T},
$$

where $l(h)$ and $a(h)$ are respectively the signed length and area of $\Gamma_{h}$ and where $r:=\min _{0 \leq t \leq T}(-h(t))$ and $R:=\max _{0 \leq t \leq T}(-h(t))$. Furthermore, the equality holds if and only if $R=r$.

Proof. Since $-h: \mathbb{R} \rightarrow \mathbb{R}$ is continuous, there exists $\left(t_{0}, t_{1}\right) \in[0, T]^{2}$ such that $r=-h\left(t_{0}\right)$ and $R=-h\left(t_{1}\right)$. We have thus

$$
(R-r)^{2}=\left(h\left(t_{1}\right)-h\left(t_{0}\right)\right)^{2}=\left(\int_{t_{0}}^{t_{1}} h^{\prime}(t) d t\right)^{2} .
$$

By the Cauchy-Schwarz inequality, we deduce that

$$
(R-r)^{2} \leq\left|t_{1}-t_{0}\right| \cdot \int_{\min \left(t_{0}, t_{1}\right)}^{\max \left(t_{0}, t_{1}\right)} h^{\prime}(t)^{2} d t \leq T \int_{0}^{T} h^{\prime}(t)^{2} d t .
$$

Now

$$
\int_{0}^{T} h^{\prime}(t)^{2} d t=2 a(h)-\int_{0}^{T} h(t)^{2} d t
$$


and again by the Cauchy-Schwarz inequality

$$
l(h)^{2}=\left(\int_{0}^{T} h(t) d t\right)^{2} \leq T \int_{0}^{T} h(t)^{2} d t .
$$

Therefore

$$
(R-r)^{2} \leq 2 T a(h)-l(h)^{2},
$$

which achieves the proof of the reversed Bonnesen inequality.

Finally, considering equality cases at each step of the reasoning, we immediately see that the equality holds if, and only if, $h$ is constant, which completes the proof.

\section{References}

[E] H.G. Eggleston, Convexity, Cambridge Tracts in Mathematics and Mathematical Physics. $\mathrm{N}^{\circ} .47$. Cambridge Univ. Press, 1958.

[F] F. Fillastre, Fuchsian convex bodies: basics of Brunn-Minkowski theory, Geom. Funct. Anal. 23, (2013), 295-333.

[G] H. Geppert, Über den Brunn-Minkowskischen Satz, Math. Z. 42 (1937), 238-254.

[G1] H. Görtler, Erzeugung stützbarer Bereiche I. Deutsche Math. 2 (1937), 454-456.

[G2] H. Görtler, Erzeugung stützbarer Bereiche II. Deutsche Math. 3 (1937), 189-200.

[L] R. Langevin, G. Levitt and H. Rosenberg, Hérissons et multihérissons (enveloppes paramétrées par leur application de Gauss). In: Singularities, Banach Center Publ. 20, Warsaw, PWN, 1988, 245-253.

[Y] Y. Martinez-Maure, De nouvelles inégalités géométriques pour les hérissons. Arch. Math. 72 (1999), 444-453.

[Y1] Y. Martinez-Maure, A fractal projective hedgehog, Demonstratio Math. 34 (2001), 59-63.

[Y6] Y. Martinez-Maure, Geometric study of Minkowski differences of plane convex bodies, Canad. J. Math. 58 (2006), 600-624.

[O] R. Osserman, Bonnesen-style isoperimetric inequalities, Am. Math. Mon. 86 (1979), 1-29 .

[S] R. Schneider, Convex Bodies: The Brunn-Minkowski Theory, 2nd expanded ed., Cambridge Univ. Press, 2014.

Y. Martinez-Maure

Institut Mathématique de Jussieu - Paris Rive Gauche 
UMR 7586 du CNRS

Bâtiment Sophie Germain

Case 7012

75205 Paris Cedex 13

France 